\title{
Intrabronchial activated protein C enhances lipopolysaccharide-induced pulmonary responses
}

\author{
Liesbeth M. Kager ${ }^{1,2}$, J. Daan de Boer ${ }^{1}$, Paul Bresser ${ }^{3,4}$, Jaring S. van der Zee ${ }^{3,4}$, \\ Sacha Zeerleder ${ }^{5,6}$, Joost C.M. Meijers ${ }^{7}$, Cornelis van 't Veer ${ }^{1,2}$ \\ and Tom van der Poll $1,2,8$
}

Affiliations: ${ }^{1}$ Center of Experimental and Molecular Medicine, Academic Medical Center/University of Amsterdam, Amsterdam, ${ }^{2}$ Center for Infection and Immunity Amsterdam, Academic Medical Center/ University of Amsterdam, Amsterdam, ${ }^{3}$ Dept of Pulmonology, Academic Medical Center/University of Amsterdam, Amsterdam, ${ }^{4}$ Dept of Pulmonology, Onze Lieve Vrouwe Gasthuis, Amsterdam, ${ }^{5}$ Dept of Hematology, Academic Medical Center/University of Amsterdam, Amsterdam, 'Dept of Immunopathology, Sanquin Research at CLB and Landsteiner Laboratory of the AMC, Amsterdam, ${ }^{7}$ Dept of Experimental Vascular Medicine, Academic Medical Center/University of Amsterdam, Amsterdam, and ${ }^{8}$ Division of Infectious Diseases, Academic Medical Center/University of Amsterdam, Amsterdam, The Netherlands.

Correspondence: L.M. Kager, Center for Experimental and Molecular Medicine, Academic Medical Center, University of Amsterdam, Meibergdreef 9, Room G2-130, 1105 AZ Amsterdam, The Netherlands. E-mail: l.m.kagerdamc.uva.nl

ABSTRACT Intravenous administration of activated protein C (APC) inhibits coagulation and inflammation in the lungs of humans and animals. Investigations in rodents demonstrated that direct intrapulmonary delivery of APC also exerts anticoagulant and anti-inflammatory effects. The effect of intrabronchial administration of recombinant human (rh)APC on lipopolysaccharide (LPS)-induced haemostatic and inflammatory alterations in the bronchoalveolar space of humans was studied.

Eight subjects received rhAPC via intrabronchial instillation by bronchoscope, while in a contralateral subsegment subjects received saline; all subjects were challenged bilaterally with LPS in the same lung subsegments. Four additional subjects received rhAPC $(75 \mu \mathrm{g})$, with saline as a control in the contralateral subsegment, while they were bilaterally "challenged" with saline. After $6 \mathrm{~h}$ a bronchoalveolar lavage was performed and coagulation and inflammatory parameters were measured.

rhAPC enhanced LPS-induced coagulation activation in the bronchoalveolar space, when compared with the control side. In addition, rhAPC amplified LPS-induced pro-inflammatory responses, as indicated by higher concentrations of cytokines and chemokines. rhAPC alone did not have procoagulant or proinflammatory effects.

Locally administered rhAPC has unexpected procoagulant and pro-inflammatory effects in LPSchallenged lung subsegments. These data argue against a role for intrapulmonary delivery of rhAPC as a treatment strategy for lung inflammatory disorders in humans.

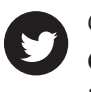

@ERSpublications

Observations made in the LPS-challenged lung question the use of rhAPC as therapy for lung inflammatory disorders http://ow.ly/ksmX7

This article has supplementary material available from www.erj.ersjournals.com

Received: April 052012 | Accepted after revision: Sept 292012 | First published online: Oct 112012

Clinical trial: This study is registered at www.clinicaltrials.gov with identifier number NCT00943267 and at www.trialregister.nl with identifier number NTR1544.

Support statement: This study was supported by research grants from ZonMW (to L.M. Kager, grant number: 92003504), the Netherlands Asthma Foundation (J.D. de Boer; project 3.2.08.009) and the Stichting BeGeTu (to L.M. Kager).

Conflict of interest: None declared.

Copyright @ERS 2013 


\section{Introduction}

The airways represent a body site in which procoagulant and anticoagulant mechanisms can be initiated and regulated locally $[1,2]$. Activated protein $\mathrm{C}$ (APC), an important anticoagulant, has been shown to have a reduced activity in the bronchoalveolar space in various lung inflammatory diseases [3-11]. Diminished APC levels may contribute to the disturbed haemostatic equilibrium in the lungs of patients suffering from these conditions, shifting the physiological anticoagulant environment into a net procoagulant state. Besides its anticoagulant effects, APC has been implicated in a variety of anti-inflammatory and/or cytoprotective effects, characterised by downregulation of pro-inflammatory pathways, upregulation of anti-inflammatory pathways and inhibition of neutrophil activity $[12,13]$.

We recently demonstrated that intrabronchial instillation of lipopolysaccharide (LPS), a constituent of the cell wall of Gram-negative bacteria, can reproduce haemostatic alterations found in inflammatory lung disorders, including a reduction in APC levels in the lungs of healthy volunteers [14, 15]. This human model of intrabronchial LPS delivery has also been used to study the in vivo effects of intravenous recombinant human (rh)APC treatment on pulmonary inflammation and coagulation $[15,16]$. Intravenous rhAPC reduced LPS-induced neutrophil accumulation in the bronchoalveolar space [16] and inhibited local activation of coagulation [15]. Concurrently, LPS-induced suppression of fibrinolysis was partially prevented by intravenous rhAPC [15].

When administered intravenously, the strong anticoagulant effects of rhAPC may contribute to serious systemic side-effects such as intracranial haemorrhages [17-20], which may be circumvented by local administration of rhAPC. Although preclinical animal studies have shown that administration of APC via the airways exerts local anti-inflammatory and anticoagulant effects [21-24], no studies exist that have evaluated the effects of direct rhAPC administration into the lungs of humans. The present study, therefore, sought to determine the feasibility of local, intrabronchial administration of rhAPC to inhibit LPS-induced lung inflammation and coagulation in humans.

\section{Methods}

\section{Study subjects and materials}

12 nonsmoking males (age $23.2 \pm 1.1$ years) were enrolled. Screening, consisting of a questionnaire, physical examination, routine blood and urine investigation, ECG and spirometry, did not reveal any abnormality. The protocol was approved by the institutional Medical Ethics Review Committee. All subjects provided written informed consent before enrolment. LPS was derived from Escherichia coli O:113 (Reference Endotoxin, CC-RE-Lot-3, National Institutes of Health, Bethesda, MD, USA). rhAPC (drotrecogin alfa (activated); Eli Lilly, Indianapolis, IN, USA) was purchased from a commercial supplier.

\section{Study design}

The study was set-up as a single-blind intervention study with a dose-escalation and a follow-up phase. A bilateral challenge of two contralateral lung subsegments was performed by intrabronchial instillation of LPS $\left(4 \mathrm{ng} \cdot \mathrm{kg}^{-1}\right)$ by bronchoscopy, diluted in $10 \mathrm{~mL}$ of sterile saline. In one lung subsegment the instillation of LPS was followed immediately by administration of rhAPC (in $10 \mathrm{~mL}$ ) in the same lung subsegment, whereas in the contralateral lung subsegment LPS administration was followed by instillation of $10 \mathrm{~mL}$ saline alone. The subjects were randomised to the left or right lung for rhAPC instillation. In the doseescalating phase of the study, five-fold increases in the rhAPC dose were intended to be given in cohorts of four subjects per dose, starting at $15 \mu \mathrm{g}$. The predefined primary end-point was to reach a $30 \%$ decrease in thrombin-antithrombin complex (TATc) concentrations in the rhAPC-treated lung $6 \mathrm{~h}$ after intrabronchial LPS challenge relative to the contralateral LPS-challenged side; this end-point was based on the capacity of intravenous rhAPC to inhibit the rise in LPS-induced TATc concentrations in bronchoalveolar lavage fluid (BALF) by approximately $30 \%$ in our previous study [15]. Per study design, the sample size of the rhAPC dose group that would reach this primary end-point would be increased to a total of 12 subjects in the follow-up phase of the study. After completion of the first two cohorts in the dose-escalation phase (15 and $75 \mu \mathrm{g} ; \mathrm{n}=4$ for each dose), we decided to stop the study because it was considered highly unlikely that the primary study end-point could be reached. Instead, four additional subjects were included who received $75 \mu \mathrm{g}$ rhAPC in one lung subsegment without previous intrabronchial LPS challenge; in these volunteers saline was given as a control in the contralateral lung subsegment.

\section{Methods}

A bilateral bronchoalveolar lavage (BAL) was performed $6 \mathrm{~h}$ post-challenge in a standardised fashion [14, 25]. Citrated and heparinised blood was taken by venipuncture before the first $(t=0 \mathrm{~h})$ and the second $(\mathrm{t}=6 \mathrm{~h})$ bronchoscopy. The assays used are described in the online supplementary material. 


\section{Statistical analysis}

Values are expressed as mean \pm SEM. Paired t-tests were used to establish significance between datasets. p-values $<0.05$ were considered statistically significant.

\section{Results}

\section{Clinical signs and systemic inflammatory response}

Instillation of LPS in two contralateral lung subsegments (combined with rhAPC in one lung segment) was well tolerated. A modest rise in body temperature was recorded $6 \mathrm{~h}$ after instillation of LPS (from $36.0 \pm 0.2^{\circ} \mathrm{C}$ at $\mathrm{t}=0 \mathrm{~h}$ to $36.6 \pm 0.1^{\circ} \mathrm{C}$ at $\left.\mathrm{t}=6 \mathrm{~h} ; \mathrm{p}<0.05\right)$. Intrabronchial LPS caused a rise in blood neutrophil count (from $2.3 \pm 0.2 \times 10^{9}$ to $6.6 \pm 0.8 \times 10^{9}$ cells $\cdot \mathrm{L}^{-1} ; \mathrm{p}<0.001$ ), while in the saline-challenged group no significant rises in neutrophils counts were seen (from $2.0 \pm 0.2 \times 10^{9}$ to $4.1 \pm 0.8 \times 10^{9}$ cells $\cdot \mathrm{L}^{-1}$ ). Finally, intrabronchial instillation of either LPS or saline, with addition of rhAPC in one lung subsegment, was not associated with changes in plasma concentrations of TATc, D-dimer, tumour necrosis factor (TNF)- $\alpha$ or interleukin (IL)-6 (data not shown).

\section{Bronchoalveolar APC concentrations}

$6 \mathrm{~h}$ after intrabronchial instillation of rhAPC, APC was measurable in all BALF samples obtained from the APC-treated side: APC levels were $0.33 \pm 0.14$ and $1.60 \pm 0.57 \mathrm{ng} \cdot \mathrm{mL}^{-1}$ in LPS-challenged subjects treated with 15 and $75 \mu \mathrm{g}$ rhAPC, respectively ( $\mathrm{p}=0.07$ and $\mathrm{p}<0.05$ for lung subsegments treated with 15 and $75 \mu \mathrm{g}$ rhAPC versus control lung subsegments; fig. $1 \mathrm{a}$ and b). In the saline-challenged group treated with $75 \mu \mathrm{g}$ rhAPC, BALF APC levels were $3.2 \pm 1.0 \mathrm{ng} \cdot \mathrm{mL}^{-1}(\mathrm{p}<0.05$; fig. 1c). APC levels in the $75 \mu \mathrm{g}$ rhAPCchallenged subsegments were not different between LPS-challenged and saline-challenged subjects. APC could not be detected in any of the control lung subsegments (i.e. those not administered with rhAPC).

\section{Intrabronchial rhAPC enhances bronchoalveolar coagulation activation after LPS challenge}

The intrabronchial concentrations of TATc have been used to determine the extent of bronchoalveolar coagulation activation in patients with acute lung injury and/or pneumonia [7, 8, 10]. Intrabronchial instillation of LPS in healthy humans is also associated with a rise in TATc levels in BALF [14, 15]. An earlier study showed that intravenously administered rhAPC could reduce LPS-induced elevations in TATc concentrations in BALF by approximately 30\% [15]. The main objective of the current study was to determine whether intrabronchially instilled rhAPC was able to inhibit LPS-induced TATc elevation in BALF and, if so, whether this anticoagulant effect was accompanied by anti-inflammatory effects. Considering the results of our previous investigation using intravenous rhAPC [15], we predefined the primary end-point of the current study as establishing the intrabronchial rhAPC dose that induced a $30 \%$ reduction in LPS-induced TATc concentrations in BALF. We chose to administer LPS in two contralateral lung subsegments, thereby eliminating inter-individual variation in LPS responsiveness and allowing every subject to serve as his own control with regard to rhAPC effects. Much to our surprise, intrabronchially
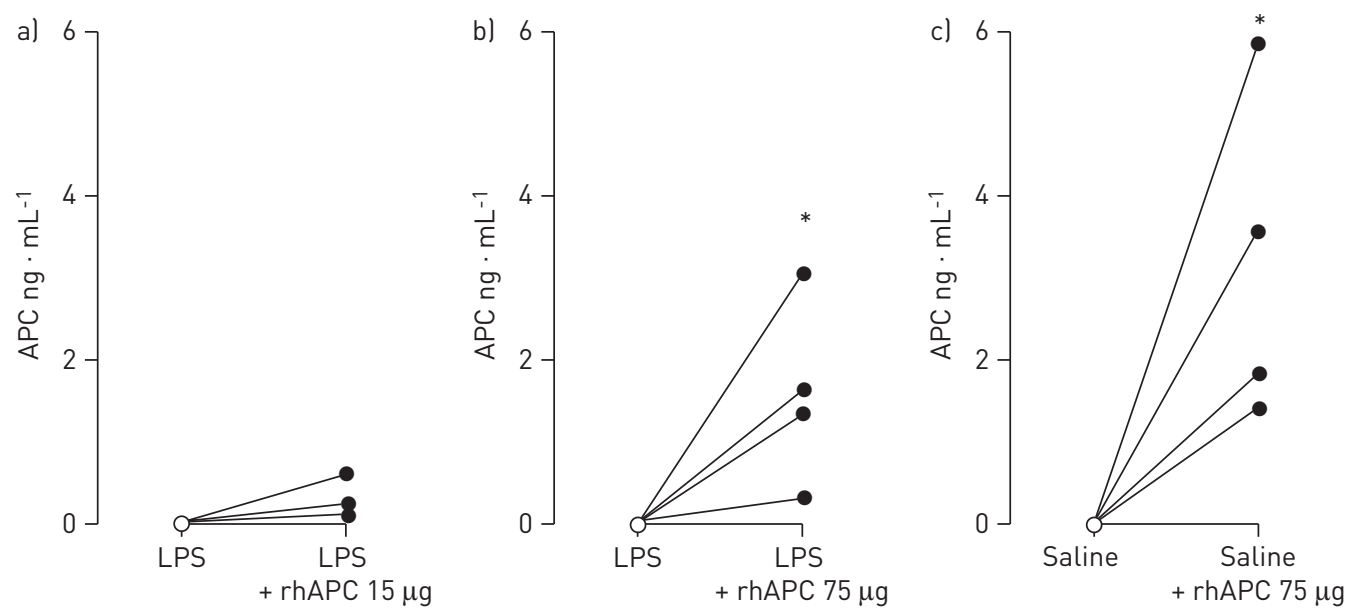

FIGURE 1 Activated protein C (APC) concentrations in bronchoalveolar lavage samples. Healthy subjects ( $\mathrm{n}=4$ per group) were challenged bilaterally in a lung subsegment via a bronchoscope with lipopolysaccharide (LPS; $4 \mathrm{ng} \cdot \mathrm{kg}^{-1} \mathrm{body}$ weight) ( $\mathrm{a}$ and b) or saline (c). Directly thereafter subjects received recombinant human ( $\mathrm{rh}$ )APC $15 \mu \mathrm{g}(\mathrm{a})$ or $75 \mu \mathrm{g}(\mathrm{b}$ and c) dissolved in $10 \mathrm{~mL}$ of saline in one subsegment and $10 \mathrm{~mL}$ of saline in the other lung subsegment. Bilateral bronchoalveolar lavage was performed $6 \mathrm{~h}$ after challenge. APC levels were undetectable in any of the control lung subsegments not administered with rhAPC. ${ }^{*}: \mathrm{p}<0.05$. 

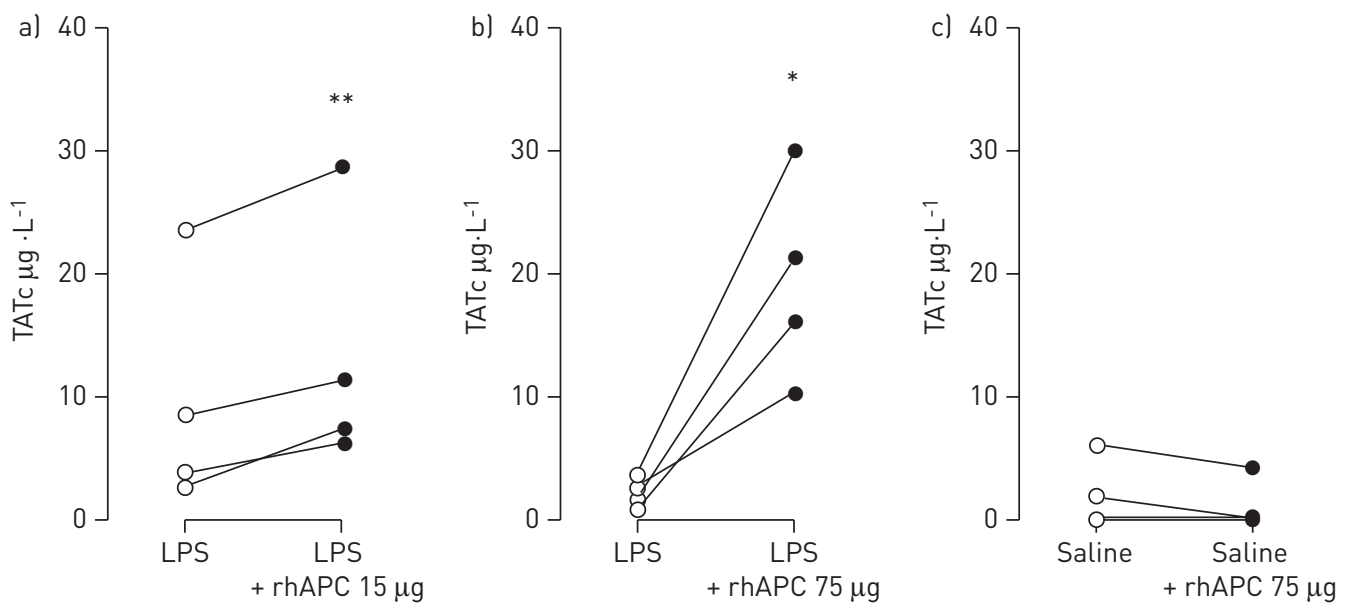

FIGURE 2 Intrabronchial instillation of recombinant human activated protein C (rhAPC) augments lipopolysaccharide (LPS)-induced coagulation in the bronchoalveolar space. Healthy subjects ( $\mathrm{n}=4$ per group) were challenged bilaterally in a lung subsegment via a bronchoscope with LPS ( $4 \mathrm{ng} \cdot \mathrm{kg}^{-1}$ body weight) (a and b) or saline (c). Directly thereafter subjects received rhAPC $15 \mu \mathrm{g}$ (a) or $75 \mu \mathrm{g}$ (b and c) dissolved in $10 \mathrm{~mL}$ of saline in one subsegment and $10 \mathrm{~mL}$ of saline in the other lung subsegment. Thrombin-antithrombin complex (TATc) levels were measured in bronchoalveolar lavage samples obtained $6 \mathrm{~h}$ after challenge. ${ }^{*}: \mathrm{p}<0.05 ;{ }^{*}: \mathrm{p}<0.01$.

instilled rhAPC, given at either $15 \mu \mathrm{g}$ or $75 \mu \mathrm{g}$, induced an increase, rather than a reduction, in BALF TATc concentrations after LPS challenge when compared with the contralateral side not treated with rhAPC (rhAPC $15 \mu \mathrm{g}$ : $13.5 \pm 5.2$ versus $9.8 \pm 4.8 \mu \mathrm{g} \cdot \mathrm{L}^{-1}$ at the contralateral side, $\mathrm{p}<0.01$, fig. $2 \mathrm{a}$; rhAPC $75 \mu \mathrm{g}$ : $19.4 \pm 4.2$ versus $2.9 \pm 0.8 \mu \mathrm{g} \cdot \mathrm{L}^{-1}$ at the contralateral side, $\mathrm{p}<0.05$, fig. $2 \mathrm{~b}$ ). As these results made it highly unlikely that we could reach our predefined end-point, we decided not to proceed with another five-fold increase in rhAPC dose but, instead, added an extra study group without LPS challenge, seeking to determine whether intrabronchial rhAPC per se elicited a procoagulant response. Thus, subjects received a bilateral challenge with saline, combined with $\operatorname{rhAPC}(75 \mu \mathrm{g})$ on one side. These studies revealed no procoagulant effect of rhAPC: BALF TATc levels were similar in lung subsegments instilled with rhAPC $\left(1.6 \pm 1.4 \mu \mathrm{g} \cdot \mathrm{L}^{-1}\right)$ when compared with the contralateral side $\left(2.4 \pm 1.2 \mu \mathrm{g} \cdot \mathrm{L}^{-1}\right.$; fig. $\left.2 \mathrm{c}\right)$. Protein C activity, total and free protein $\mathrm{S}$, soluble thrombomodulin, protein $\mathrm{C}$ inhibitor and von Willebrand factor antigen concentrations were all below detection limits in BALF.
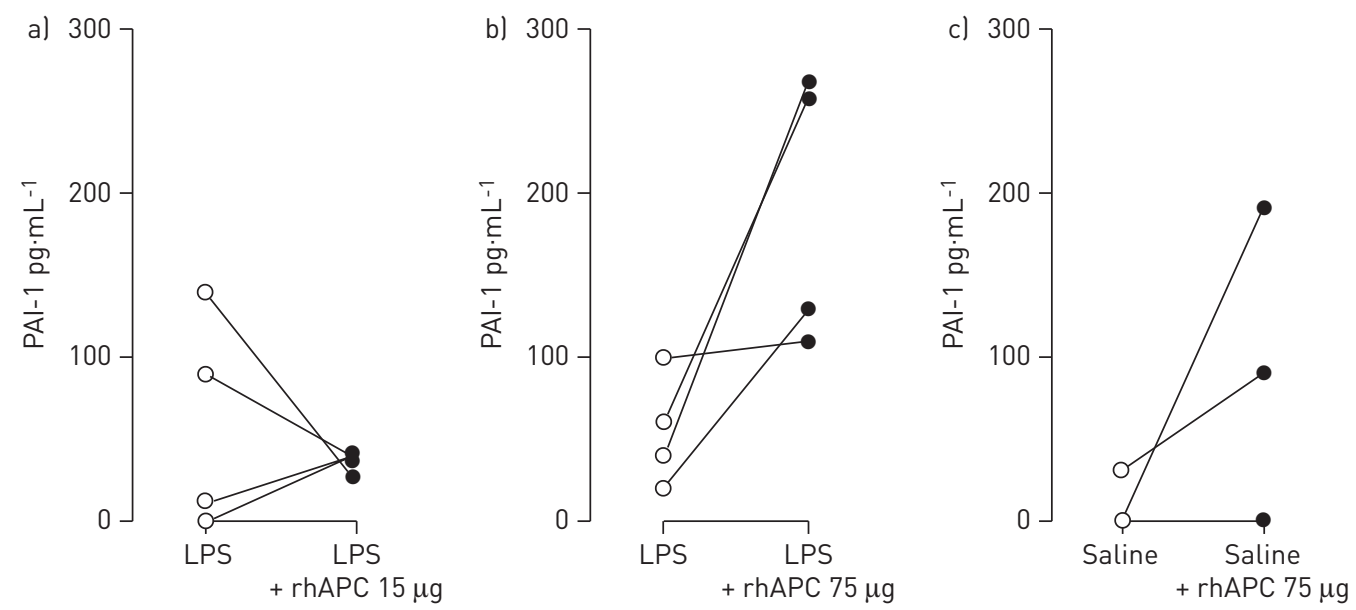

FIGURE 3 Impact of intrabronchial recombinant human activated protein C (rhAPC) on lipopolysaccharide (LPS)induced release of plasminogen activator inhibitor type I in the bronchoalveolar space. Healthy subjects ( $\mathrm{n}=4$ per group) were challenged bilaterally in a lung subsegment via a bronchoscope with LPS ( $4 \mathrm{ng} \cdot \mathrm{kg}^{-1}$ body weight) ( $\mathrm{a}$ and b) or saline (c). Directly thereafter subjects received rhAPC $15 \mu \mathrm{g}$ (a) or $75 \mu \mathrm{g}$ (b and c) dissolved in $10 \mathrm{~mL}$ of saline in one subsegment and $10 \mathrm{~mL}$ of saline in the other lung subsegment. Plasminogen activator inhibitor type 1 (PAI-1) levels were measured in bronchoalveolar lavage samples obtained $6 \mathrm{~h}$ after challenge. 

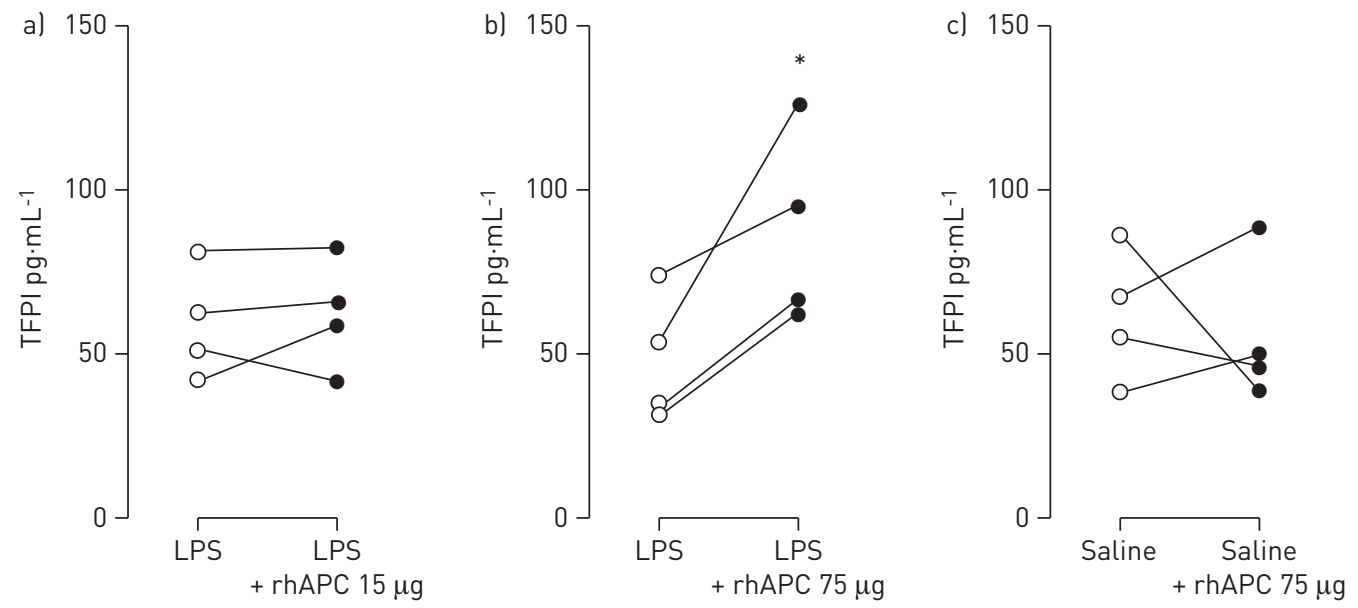

FIGURE 4 Impact of intrabronchial recombinant human activated protein C (rhAPC) on lipopolysaccharide (LPS)induced release of tissue-factor pathway inhibitor in the bronchoalveolar space. Healthy subjects ( $\mathrm{n}=4$ per group) were challenged bilaterally in a lung subsegment via a bronchoscope with LPS ( $4 \mathrm{ng} \cdot \mathrm{kg}^{-1}$ body weight) (a and b) or saline (c). Directly thereafter subjects received rhAPC $15 \mu \mathrm{g}$ (a) or $75 \mu \mathrm{g}$ (b and c) dissolved in $10 \mathrm{~mL}$ of saline in one subsegment and $10 \mathrm{~mL}$ of saline in the other lung subsegment. Tissue factor pathway inhibitor (TFPI) levels were measured in bronchoalveolar lavage samples obtained $6 \mathrm{~h}$ after challenge. ${ }^{*}: \mathrm{p}<0.05$.

\section{Effect of intrabronchial rhAPC on fibrinolysis after LPS challenge}

Evidence derived from in vitro investigations indicates that APC may stimulate fibrinolysis by inhibiting plasminogen activator inhibitor type 1 (PAI-1) [26]. We measured levels of PAI-1, tissue-type plasminogen activator (tPA) and plasmin- $\alpha 2$-antiplasmin complexes. While intrabronchial rhAPC given at a dose of $15 \mu \mathrm{g}$ did not influence LPS-induced PAI-1 release in BALF ( $\mathrm{p}=0.57$; fig. 3a), rhAPC administered at $75 \mu \mathrm{g}$ increased LPS-induced PAI-1 BALF levels in three out of the four subjects $\left(192.5 \pm 42.1\right.$ versus $55.0 \pm 17.1 \mathrm{pg} \cdot \mathrm{mL}^{-1}$ at the contralateral side; $\mathrm{p}=0.07$; fig. $3 \mathrm{~b}$ ). Notably, intrabronchial rhAPC also tended to increase PAI-1 levels in BALF from subjects who did not receive LPS $\left(70.0 \pm 45.3\right.$ versus $7.5 \pm 7.5 \mathrm{pg} \cdot \mathrm{mL}^{-1}$ at the contralateral side; $\mathrm{p}=0.26$; fig. 3c). Neither LPS nor rhAPC influenced BALF concentrations of plasmin- $\alpha 2$-antiplasmin complexes, whereas tPA levels were below detection limit in all BALF samples (data not shown).

Intrabronchial rhAPC is associated with enhanced concentrations of tissue factor pathway inhibitor after LPS challenge

Recent data suggest that APC can have a procoagulant effect by virtue of its proteolytic activity. APC was reported to shed the Kunitz-1 domain from tissue factor pathway inhibitor (TFPI), resulting in increased tissue factor activity and, consequently, coagulation activation [27]. We measured levels of TFPI in BALF. While intrabronchial rhAPC given at a dose of $15 \mu \mathrm{g}$ did not influence LPS-induced TFPI release in BALF (fig. 4a), rhAPC administered at $75 \mu \mathrm{g}$ increased LPS-induced TFPI BALF levels in all four subjects ( $48.1 \pm 9.9$ versus $87.9 \pm 14.8 \mathrm{pg} \cdot \mathrm{mL}^{-1}$ at the contralateral side; $\mathrm{p}<0.05$; fig. $\left.4 \mathrm{~b}\right)$. Intrabronchial rhAPC did not change levels of TFPI in BALF from subjects who did not receive LPS (fig. 4c).

\section{Intrabronchial rhAPC increases leukocyte recruitment after LPS administration}

Intravenous rhAPC has been reported to reduce neutrophil recruitment into the bronchoalveolar space upon intrabronchial instillation of LPS in healthy humans [16]. In contrast, intrabronchial rhAPC treatment increased the total number of white blood cells in BALF $6 \mathrm{~h}$ after bilateral LPS challenge, although the differences with the contralateral control side did not reach statistical significance (APC $15 \mu \mathrm{g}$ : $33.6 \pm 14.6 \times 10^{4}$ versus $18.6 \pm 4.1 \times 10^{4}$ cells $\cdot \mathrm{mL}^{-1}$ at the contralateral side, $\mathrm{p}=0.25$, fig. $5 \mathrm{a}$; APC $75 \mu \mathrm{g}$ : $52.0 \pm 22.4 \times 10^{4}$ versus $11.1 \pm 1.5 \times 10^{4}$ cells $\cdot \mathrm{mL}^{-1}$ at the contralateral side, $\mathrm{p}=0.13$, fig. $5 \mathrm{~b}$ ). The rhAPCinduced increase in total cell number in lung subsegments challenged with LPS was due to a rise in the number of neutrophils (APC $15 \mu \mathrm{g}$ : $16.8 \pm 9.0 \times 10^{4}$ versus $7.5 \pm 2.7 \times 10^{4}$ neutrophils $\cdot \mathrm{mL}^{-1}, \mathrm{p}=0.25$, fig. $5 \mathrm{~d}$; APC $75 \mu \mathrm{g}: 34.1 \pm 15.8 \times 10^{4}$ versus $1.5 \pm 0.6 \times 10^{4}$ neutrophils $\cdot \mathrm{mL}^{-1}, \mathrm{p}=0.13$, fig. $\left.5 \mathrm{e}\right)$. Intrabronchial rhAPC did not affect cell counts in lung subsegments not challenged with LPS (fig. $5 \mathrm{c}$ and $\mathrm{f}$ ).

\section{Intrabronchial rhAPC enhances the local release of several cytokines and chemokines after LPS} instillation

To investigate the effect of local rhAPC administration on LPS-induced inflammatory mediator release we measured BALF concentrations of 48 cytokines, chemokines and growth factors. The results of these 

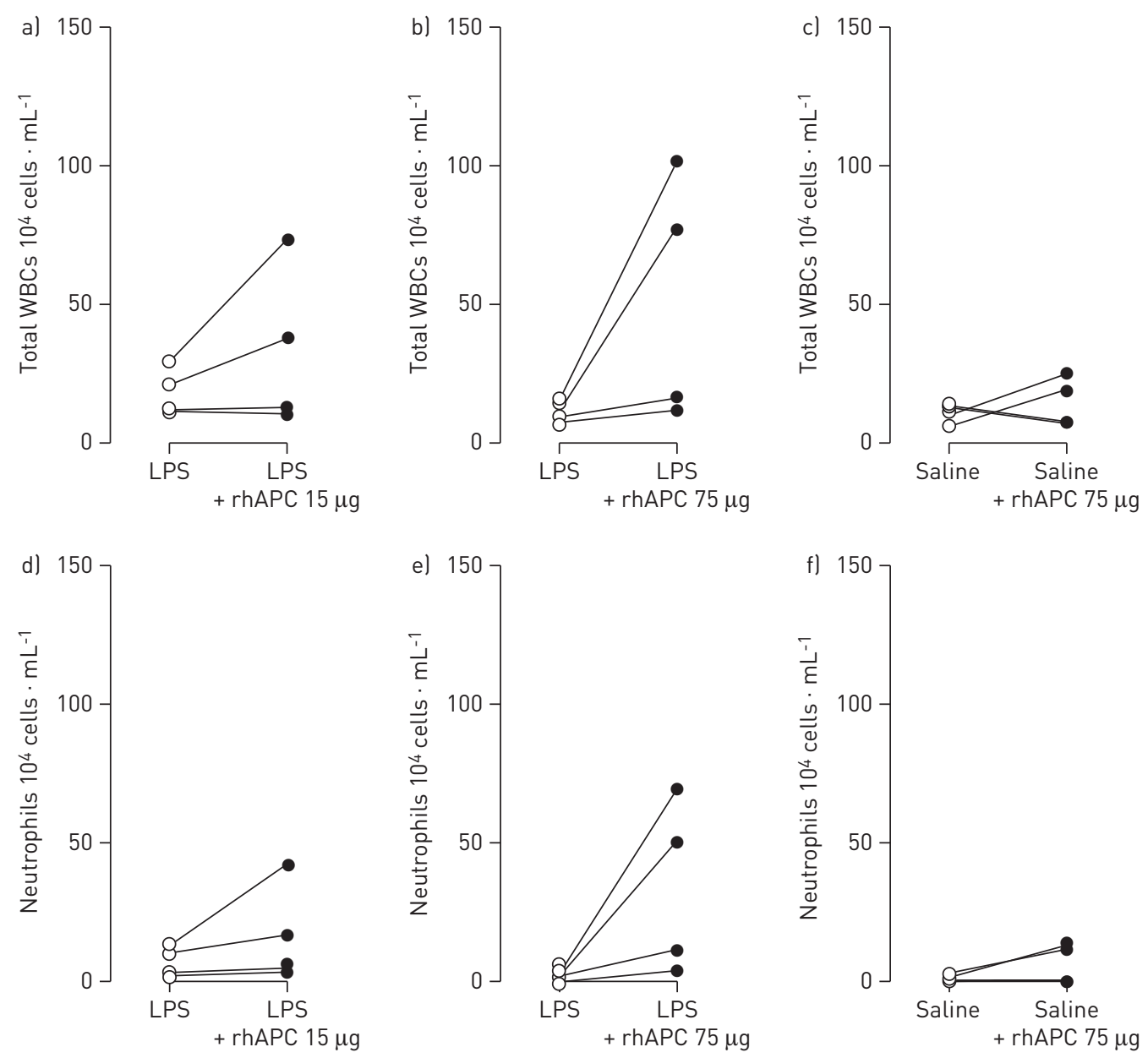

FIGURE 5 Impact of intrabronchial recombinant human activated protein C (rhAPC) on the lipopolysaccharide (LPS)induced increase in total leukocyte and neutrophil counts in the bronchoalveolar space. Healthy subjects ( $\mathrm{n}=4$ per group) were challenged bilaterally in a lung subsegment via a bronchoscope with LPS ( $4 \mathrm{ng} \cdot \mathrm{kg}^{-1}$ body weight) ( $\mathrm{a}$ and b) or saline (c). Directly thereafter subjects received rhAPC $15 \mu \mathrm{g}$ (a) or $75 \mu \mathrm{g}$ (b and c) dissolved in $10 \mathrm{~mL}$ of saline in one subsegment and $10 \mathrm{~mL}$ of saline in the other lung subsegment. White blood cell counts (WBCs) and neutrophil counts were determined in bronchoalveolar lavage samples obtained $6 \mathrm{~h}$ after challenge.

analyses are shown in online supplementary table S1. In the lower rhAPC dose cohort $(15 \mu \mathrm{g}), \mathrm{rhAPC}$ significantly enhanced LPS-induced release of IL-6, CCL8 and CXCL6 while not influencing the levels of other mediators (online supplementary table S1). In the rhAPC $75 \mu \mathrm{g}$ cohort, rhAPC significantly enhanced LPS-induced TNF- $\alpha$, CCL4, CCL8 and TNF-related apoptosis-inducing ligand (TRAIL) concentrations (fig. 6 and online supplementary table S1). In addition, IL-1 $\beta$, CCL14a, CCL20, CXCL7, CXCL9 and CXCL11 showed a strong trend toward higher levels in this higher rhAPC dose cohort $(\mathrm{p}=0.052, \mathrm{p}=0.081$, $p=0.078, p=0.075, p=0.092$ and $p=0.074$, respectively; fig. 6 and online supplementary table S1). rhAPC given at $75 \mu \mathrm{g}$ without LPS did not induce the release of inflammatory mediators in the lung, with the exception of a modest rise in CCL15 concentrations (online supplementary table S1). Taken together, these results show that intrabronchial instillation of rhAPC in LPS-challenged lung subsegments enhanced the release of multiple pro-inflammatory mediators.

\section{Discussion}

Several preclinical studies have shown that APC may protect the lung from injury caused by inflammation [28]. Moreover, intravenous administration of rhAPC was reported to exert anticoagulant and antiinflammatory effects in the bronchoalveolar space of healthy humans challenged with LPS in a lung subsegment by bronchoscope $[15,16]$. Animal studies demonstrated that intrabronchially instilled APC exerts local anticoagulant and anti-inflammatory effects [21-24, 29-32]. Considering the bleeding risk associated with intravenous APC, we here for the first time investigated the potential of intrabronchial rhAPC administration to inhibit coagulation and inflammation in the human lung, arguing that this route 

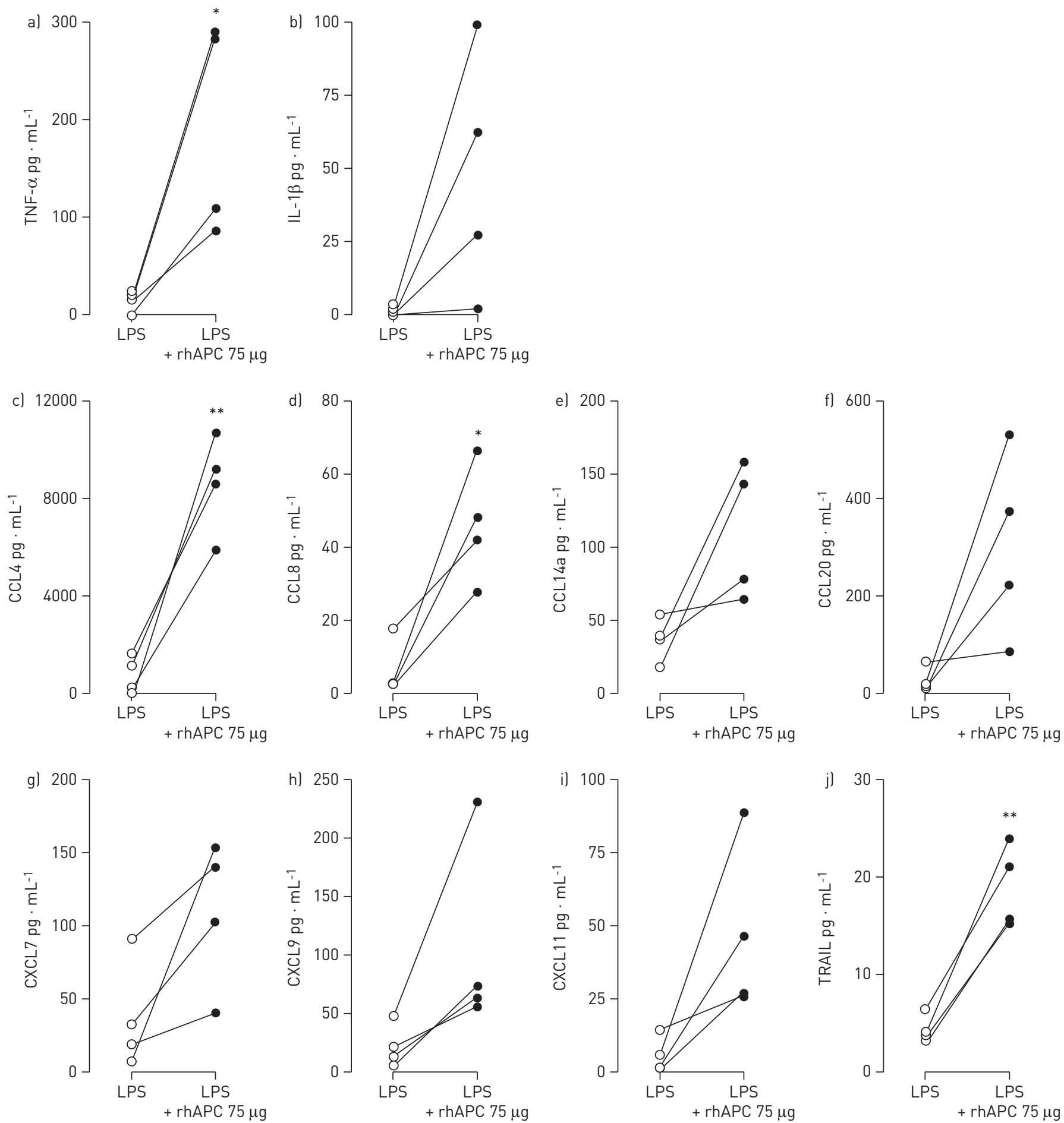

FIGURE 6 Intrabronchial instillation of recombinant human activated protein C (rhAPC) augments lipopolysaccharide (LPS)-induced release of inflammatory mediators in the bronchoalveolar space. Healthy subjects $(\mathrm{n}=4$ per group) were challenged bilaterally in a lung subsegment via a bronchoscope with LPS ( $4 \mathrm{ng} \cdot \mathrm{kg}^{-1}$ body weight). Directly thereafter subjects received rhAPC $75 \mu \mathrm{g}$ dissolved in $10 \mathrm{~mL}$ of saline in one subsegment and $10 \mathrm{~mL}$ of saline in the other lung subsegment. Mediator levels were measured in bronchoalveolar lavage samples obtained $6 \mathrm{~h}$ after challenge. TNF: tumour necrosis factor; IL: interleukin; TRAIL: TNF-related apoptosis-inducing ligand. ${ }^{*}: \mathrm{p}<0.05 ;{ }^{* *}: \mathrm{p}<0.01$.

of delivery would circumvent serious side-effects [17-20]. For this we used the established human model of lung inflammation induced by intrabronchial instillation of LPS via a bronchoscope [14-16, 25]. The main finding of our study was remarkable: intrabronchially administered rhAPC induced a procoagulant and pro-inflammatory response in lung subsegments challenged with LPS rather than the expected inhibitory 
effects on coagulation and inflammation. Our results from subjects not challenged with LPS via the airways further suggest that rhAPC augments LPS effects in the human lung, while not eliciting detectable inflammation or coagulation per se.

Our study had a small sample size. As a consequence, although rhAPC clearly enhanced the LPS-induced release of biomarkers of coagulation and inflammation, the differences with the placebo control side often did not reach statistical significance. However, the current study was designed to demonstrate an inhibitory effect of rhAPC on bronchoalveolar coagulation and inflammation. After completion of the rhAPC $75 \mu \mathrm{g}$ cohort, we considered it unlikely that the predefined end-point (i.e. a 30\% reduction in BALF TATc levels) could be reached. Therefore, we decided to prematurely terminate the study. In addition, we considered it not ethical to enlarge the sample size of our cohorts in order to strengthen the statistical power of our study, on the basis that procoagulant and pro-inflammatory effects of locally administered rhAPC would not be of clinical value for patient groups and wishing to avoid exposing additional healthy subjects to invasive procedures.

In the earlier study, in which intravenous rhAPC was found to inhibit coagulation and neutrophil influx in the same human LPS-induced lung inflammation model $[15,16]$, APC concentrations in BALF were approximately $15 \mathrm{ng} \cdot \mathrm{mL}^{-1}$ as measured $2 \mathrm{~h}$ after discontinuation of the rhAPC infusion $\left(24 \mu \mathrm{g}^{-\mathrm{kg}^{-1}} \cdot \mathrm{h}^{-1}\right)$ [15]. Although BALF APC levels detected after intravenous rhAPC infusion cannot be readily translated to a feasible rhAPC dose to be administered in the bronchoalveolar space, we conservatively started the doseescalation study with $15 \mu \mathrm{g}$ of intrabronchial rhAPC, taking into account the dilution caused by the BAL procedure and an estimated recovery of 50-60\%. BALF APC levels measured in the current study, $6 \mathrm{~h}$ after instillation, were considerably lower than in the previous study using intravenous APC administration [15] (mean levels of $0.33 \mathrm{ng} \cdot \mathrm{mL}^{-1}$ and $1.60 \mathrm{ng} \cdot \mathrm{mL}^{-1}$ in the rhAPC $15 \mu \mathrm{g}$ and $75 \mu \mathrm{g}$ cohorts, respectively). Arguably, BALF APC concentrations were higher directly after rhAPC administration. Clearance of APC from the bronchoalveolar space is difficult to investigate in humans in light of the invasive procedure needed; nonetheless, evidence suggests that APC is slowly cleared from the alveolar compartment. Indeed, while the majority of patients with sepsis who are treated with intravenous rhAPC do not have detectable APC in plasma $2 \mathrm{~h}$ after discontinuation of the infusion [33], APC could still be recovered from the BALF of healthy humans in whom a similar rhAPC infusion was stopped $2 \mathrm{~h}$ earlier [15]. Hence, although BALF APC concentrations measured in the present (intrabronchial rhAPC) and previous (intravenous rhAPC) [15] investigation cannot be compared easily, these combined data suggest that the APC levels achieved in BALF here were likely within the same or lower range than those attained after intravenous rhAPC infusion.

How can the apparently differential effects of intravenous rhAPC [15] and intrabronchial rhAPC in LPSinduced human lung inflammation be explained? APC can exert anti-inflammatory, anti-apoptotic and barrier protective signals in endothelial cells via protease-activated receptor (PAR) 1 by a mechanism that requires binding of APC to the endothelial cell protein $C$ receptor (EPCR) [34]. Cytoprotective antiinflammatory APC-PAR1 signalling has also been demonstrated in other cell types, in particular dendritic cells (EPCR dependent [35]) and macrophages (CD11/CD18 dependent $[35,36])$. Furthermore, APC directly binds to activated $\alpha_{3} \beta_{1}, \alpha_{5} \beta_{1}$ and $\alpha_{V} \beta_{3}$ integrins and this interaction was essential for APC-induced inhibition of neutrophil extravasation into the bronchoalveolar space of mice [37]. These "cytoprotective" and/or anti-inflammatory APC effects in vivo likely depend on which cell types are affected and thereby on the route of rhAPC administration. Furthermore, the distribution of receptors involved in these APC effects may vary in different body compartments. In addition, recent data suggest that APC can have a procoagulant effect by virtue of its proteolytic activity; APC was reported to shed the Kunitz-1 domain from TFPI, resulting in increased tissue factor activity and, consequently, coagulation activation [27]. In this study we measured TFPI levels in BALF and showed that administration of rhAPC in the $75 \mu \mathrm{g}$ cohort was associated with significantly enhanced TFPI levels. We hypothesise that increased levels of TFPI may have been a result of the pro-inflammatory state due to rhAPC administration. It should be noted that the TFPI assay used detects both full-length and cleaved TFPI. Hence, it is possible that the elevated TFPI levels were the result of cleavage of the intact protein by rhAPC, thereby contributing to a procoagulant effect. We do not have a clear explanation for the discrepancy between our current findings and earlier reports on the effect of intrapulmonary delivery of APC in mice [21-24], although differences in the exact method of administration, the dose and species studied may be involved.

Our study does not provide a clear mechanism underlying the unexpected pro-inflammatory and procoagulant effect of rhAPC in the human lung. We tried to reproduce the effects of APC in a mixed cell culture system using respiratory epithelial cells (A549) and either primary human alveolar macrophages or macrophage-like THP-1 cells but were unsuccessful (data not shown).

In conclusion, this is the first study on the effects of intrabronchially administered rhAPC in a controlled lung inflammation model in humans. Our data show that locally administered rhAPC has procoagulant and 
pro-inflammatory effects in lung segments exposed to LPS. These data argue against a role for intrapulmonary delivery of rhAPC as a treatment strategy for lung inflammatory disorders in humans.

\section{Acknowledgements}

The authors thank A.F. de Vos, D. Kruijswijk and D.C. Blok (all Center for Experimental and Molecular Medicine, Academic Medical Center/University of Amsterdam, Amsterdam, The Netherlands) for their assistance during the study days, G. van Mierlo (Dept of Immunopathology, Sanquin Research at CLB, Amsterdam, The Netherlands), W.F. Kopatz, M.A. Weijne, L.M. Leverink and J.A. Marquart (all Dept of Experimental Vascular Medicine, Academic Medical Center/ University of Amsterdam, The Netherlands) for assistance in performing the coagulation measurements and E.M. Kemper, in-hospital pharmacist (Dept of Pharmacology, Academic Medical Center/University of Amsterdam, The Netherlands) for assistance in preparation of the study medication.

\section{References}

1 Levi M, Schultz MJ, Rijneveld AW, et al. Bronchoalveolar coagulation and fibrinolysis in endotoxemia and pneumonia. Crit Care Med 2003; 31: S238-S242.

2 Wygrecka M, Jablonska E, Guenther A, et al. Current view on alveolar coagulation and fibrinolysis in acute inflammatory and chronic interstitial lung diseases. Thromb Haemost 2008; 99: 494-501.

Choi G, Schultz MJ, Levi M, et al. Protein C in pneumonia. Thorax 2005; 60: 705-706.

Hataji O, Taguchi O, Gabazza EC, et al. Activation of protein C pathway in the airways. Lung 2002; 180: 47-59. Imokawa S, Sato A, Hayakawa $\mathrm{H}$, et al. Tissue factor expression and fibrin deposition in the lungs of patients with idiopathic pulmonary fibrosis and systemic sclerosis. Am J Respir Crit Care Med 1997; 156: 631-636.

6 Schouten M, Van De Pol MA, Levi M, et al. Early activation of coagulation after allergen challenge in patients with allergic asthma. J Thromb Haemost 2009; 7: 1592-1594.

7 Schultz MJ, Millo J, Levi M, et al. Local activation of coagulation and inhibition of fibrinolysis in the lung during ventilator associated pneumonia. Thorax 2004; 59: 130-135.

8 Choi G, Schultz MJ, van Till JW, et al. Disturbed alveolar fibrin turnover during pneumonia is restricted to the site of infection. Eur Respir J 2004; 24: 786-789.

9 Ware LB, Fang X, Matthay MA. Protein C and thrombomodulin in human acute lung injury. Am J Physiol Lung Cell Mol Physiol 2003; 285: L514-L521.

10 Gunther A, Mosavi P, Heinemann S, et al. Alveolar fibrin formation caused by enhanced procoagulant and depressed fibrinolytic capacities in severe pneumonia. Comparison with the acute respiratory distress syndrome. Am J Respir Crit Care Med 2000; 161: 454-462.

11 Arndt PG, Young SK, Worthen GS. Regulation of lipopolysaccharide-induced lung inflammation by plasminogen activator inhibitor-1 through a JNK-mediated pathway. J Immunol 2005; 175: 4049-4059. Mosnier LO, Zlokovic BV, Griffin JH. The cytoprotective protein C pathway. Blood 2007; 109: 3161-3172. Weiler H. Regulation of inflammation by the protein C system. Crit Care Med 2010; 38: Suppl. 2, S18-S25. Hoogerwerf JJ, de Vos AF, Levi M, et al. Activation of coagulation and inhibition of fibrinolysis in the human lung on bronchial instillation of lipoteichoic acid and lipopolysaccharide. Crit Care Med 2009; 37: 619-625.

15 van der Poll T, Levi M, Nick JA, et al. Activated protein C inhibits local coagulation after intrapulmonary delivery of endotoxin in humans. Am J Respir Crit Care Med 2005; 171: 1125-1128.

16 Nick JA, Coldren CD, Geraci MW, et al. Recombinant human activated protein C reduces human endotoxininduced pulmonary inflammation via inhibition of neutrophil chemotaxis. Blood 2004; 104: 3878-3885.

17 Bernard GR, Vincent JL, Laterre PF, et al. Efficacy and safety of recombinant human activated protein C for severe sepsis. N Engl J Med 2001; 344: 699-709.

18 Vincent JL, Bernard GR, Beale R, et al. Drotrecogin alfa (activated) treatment in severe sepsis from the global openlabel trial ENHANCE: further evidence for survival and safety and implications for early treatment. Crit Care Med 2005; 33: 2266-2277.

19 Abraham E, Laterre PF, Garg R, et al. Drotrecogin alfa (activated) for adults with severe sepsis and a low risk of death. N Engl J Med 2005; 353: 1332-1341.

20 Marti-Carvajal AJ, Sola I, Lathyris D, et al. Human recombinant activated protein C for severe sepsis. Cochrane Database Syst Rev 2011; 4: CD004388.

21 Hofstra JJ, Vlaar AP, Cornet AD, et al. Nebulized anticoagulants limit pulmonary coagulopathy, but not inflammation, in a model of experimental lung injury. J Aerosol Med Pulm Drug Deliv 2010; 23: 105-111.

22 Cornet $\mathrm{AD}$, Hofstra JJ, Vlaar AP, et al. Nebulized anticoagulants limit coagulopathy but not inflammation in pseudomonas aeruginosa-induced pneumonia in rats. Shock 2011; 36: 417-423.

23 Slofstra SH, Groot AP, Maris NA, et al. Inhalation of activated protein C inhibits endotoxin-induced pulmonary inflammation in mice independent of neutrophil recruitment. Br J Pharmacol 2006; 149: 740-746.

24 Maniatis NA, Letsiou E, Orfanos SE, et al. Inhaled activated protein C protects mice from ventilator-induced lung injury. Crit Care 2010; 14: R70.

25 Hoogerwerf JJ, de Vos AF, Bresser P, et al. Lung inflammation induced by lipoteichoic acid or lipopolysaccharide in humans. Am J Respir Crit Care Med 2008; 178: 34-41.

26 Bajzar L, Nesheim ME, Tracy PB. The profibrinolytic effect of activated protein C in clots formed from plasma is TAFI-dependent. Blood 1996; 88: 2093-2100.

27 Schuepbach RA, Velez K, Riewald M. Activated protein C up-regulates procoagulant tissue factor activity on endothelial cells by shedding the TFPI Kunitz 1 domain. Blood 2011; 117: 6338-6346.

28 Suzuki K, Gabazza EC, Hayashi T, et al. Protective role of activated protein C in lung and airway remodeling. Crit Care Med 2004; 32: Suppl. 5, S262-S265.

29 Yuda H, Adachi Y, Taguchi O, et al. Activated protein $\mathrm{C}$ inhibits bronchial hyperresponsiveness and Th2 cytokine expression in mice. Blood 2004; 103: 2196-2204.

30 Yasui H, Gabazza EC, Tamaki S, et al. Intratracheal administration of activated protein C inhibits bleomycininduced lung fibrosis in the mouse. Am J Respir Crit Care Med 2001; 163: 1660-1668. 
31 Shimizu S, Gabazza EC, Taguchi O, et al. Activated protein $\mathrm{C}$ inhibits the expression of platelet-derived growth factor in the lung. Am J Respir Crit Care Med 2003; 167: 1416-1426.

32 Waerhaug $\mathrm{K}$, Kuzkov VV, Kuklin VN, et al. Inhaled aerosolised recombinant human activated protein C ameliorates endotoxin-induced lung injury in anaesthetised sheep. Crit Care 2009; 13: R51.

33 Macias WL, Dhainaut JF, Yan SC, et al. Pharmacokinetic-pharmacodynamic analysis of drotrecogin alfa (activated) in patients with severe sepsis. Clin Pharmacol Ther 2002; 72: 391-402.

34 Danese S, Vetrano S, Zhang L, et al. The protein C pathway in tissue inflammation and injury: pathogenic role and therapeutic implications. Blood 2010; 115: 1121-1130.

35 Kerschen E, Hernandez I, Zogg M, et al. Activated protein C targets $\mathrm{CD} 8^{+}$dendritic cells to reduce the mortality of endotoxemia in mice. J Clin Invest 2010; 120: 3167-3178.

36 Cao C, Gao Y, Li Y, et al. The efficacy of activated protein C in murine endotoxemia is dependent on integrin CD11b. J Clin Invest 2010; 120: 1971-1980.

37 Elphick GF, Sarangi PP, Hyun YM, et al. Recombinant human activated protein C inhibits integrin-mediated neutrophil migration. Blood 2009; 113: 4078-4085. 